\title{
Changes in Otolaryngology Residency During COVID-19 Times
}

\author{
Vinoth Manimaran ${ }^{1}$ (D) Somu Lakshmanan ${ }^{1}$ (D) Thirunavukkarasu Palanisamy ${ }^{1}$ (I) \\ Nethra Dinakaran ${ }^{1}$ (D) Ajay kumar Prabakar ${ }^{1}$ (B)
}

Received: 4 November 2020/Accepted: 15 May 2021/Published online: 18 June 2021

(C) Association of Otolaryngologists of India 2021

\begin{abstract}
Impact of Severe Acute Respiratory Syndrome Coronavirus 2 (SARS-CoV-2) on resident training programs is little known and even lesser studied. Residents work simultaneously in Corona virus induced disease (COVID) and non-COVID environments. Partial to complete shutdown of elective services have affected resident training significantly. This article describes changes brought about in Otolaryngology training program in a medical college hospital located in south India.
\end{abstract}

Keywords Otolaryngology $\cdot$ Residency $\cdot$ COVID-19

\section{Introduction}

Severe Acute Respiratory Syndrome Coronavirus 2 (SARS-CoV-2) has caused tremendous burden on our health care system [1]. As frontline workers, our focus has shifted towards healthcare and saving as many lives as possible. Little attention has been paid to the drastic

Vinoth Manimaran

vinomb88@gmail.com

Somu Lakshmanan

somu_11496@yahoo.com

Thirunavukkarasu Palanisamy

druroboricforms@gmail.com

Nethra Dinakaran

nethra.dinakaran@gmail.com

Ajay kumar Prabakar

ajaykumarprabakar@yahoo.in

1 Department of Otolaryngology, Head and Neck Surgery, Sri Ramachandra Institute of Higher Education and Research, Chennai 600116, India changes occurring in resident training programs across the world. Residents of all specialities are involved in the management of Corona virus Induced Disease-19 (COVID19) patients amidst their routine core work [2]. The residents' training program has suffered a great setback due to shut down of outpatient department and elective surgeries [3]. With the pandemic still raging, changes in residency program are essential to achieve the desirable competency for outgoing residents. This article describes the changes in the otolaryngology training program implemented in a tertiary care teaching hospital of south India.

\section{Resident's Academic program}

Institutional Ethics Committee approval obtained (IEC-NI/ 20/NOV/89/100). Resident programs are characterized by a fine balance between clinical exposure and didactic academic sessions. Due to COVID-19 the classes have moved from bedside clinics to lectures. Online classes can be conducted from remote locations and recorded for future reference. It can be viewed later by students who are unable to attend live sessions. However, we felt that the virtual interaction between teachers and students was lesser than in-person interaction. The number of didactic academic sessions have increased, due to reduced clinical work. We have also observed an increase in attendance of post graduate students and teaching faculty members in COVID times. This might be due to the fact that the faculty members engaged in operation theatre and emergency services may have missed the classes during pre-COVID times. The author's institution has a dedicated e-learning platform, where all classes are recorded and uploaded, made available for future reference. This has enabled the postgraduates to watch these sessions later even if they have missed the live session. 
Table 1 Changes in the academic sessions during COVID times

\begin{tabular}{ll}
\hline Sessions increased & Sessions decreased \\
\hline Resident and faculty Lectures & Clinical exposure in core subject \\
Temporal bone dissection & In person interaction with teachers \\
Research time & Case discussions \\
Interdepartmental CME &
\end{tabular}

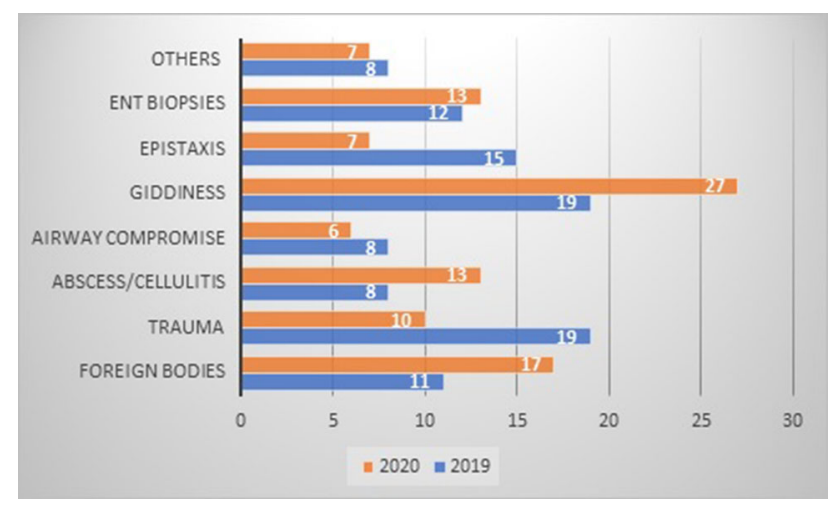

Fig. 1 Distribution of cases (\%) attended in Emergency department in 2019 and 2020

Hands on temporal bone training is critical for performing otological surgeries. Each resident was allotted $2 \mathrm{~h} /$ week for dissection in pre-COVID times. However due to reduced clinical work, the dissection time has been increased to $4 \mathrm{~h} /$ week. The dissected bones are photographed and uploaded in the e-learning platform. This can be accessed by all faculty members and the feedback of dissections obtained. Online Continuing Medical Examination (CME) programs involving guest faculty members from closely related departments likely radiology, oncology and plastic surgery have been introduced.

Research is an integral part of resident training. COVID19 has increased the time for research among the faculty members and students. The department had published 3, submitted 14 and proposed 5 research manuscripts which is twice as much as the previous academic year. This could be attributed to increased time available to obtain, process and publish data.

Scientific paper and poster presentations at national conferences are mandatory in the Indian otolaryngology resident training program. With a nationwide lockdown, these paper presentations have become difficult for residents. As most of conferences have moved online, the presentations have also moved online, where the trainees are asked to submit their presentations well in advance along with voice recordings. Though this might help in fulfilling their competencies for residency, online presentations lack the experience of direct interaction with guest faculty members and observing presentations of other institutes.

Activities like student seminars, journal club and case presentations play a major role in post graduate teaching. Of these, case presentations have suffered a major setback due to shutdown of outpatient and inpatient services of non-emergency nature and COVID restrictions in place. As most inpatient admissions are for emergency procedures, the discussion on core topics have become less. As a result, most universities across the country have conducted the final exams by introducing virtual case scenarios and OSCE (Objective Structured Clinical Examination). As the near future of real case presentations look bleak, we are left with only virtual case presentations. Case based discussions help to assess candidate's clinical application of his knowledge. So, we collected case records of patients from MRD (Medical Records Division) section who were of clinical interest. History was noted down from case sheets and images were collected from endoscopy and radiology software. Though the virtual case scenarios lack the clinical skill assessment, it helped us to continue the case discussion forum. Table 1 shows the sessions in residency programs which have increased and decreased.

\section{Patient Oriented Services}

As a response to the ongoing pandemic, hospitals across the country have formed COVID response teams inducting medical professionals across disciplines. As a result, residents deal with COVID and non-COVID patients working in shifts around the clock. Their clinical exposure to core subject has been greatly compromised. Elective surgical and outpatient experiences are greatly affected because of the shutdown of these services. We retrospectively audited the Emergency calls received and compared with last year data in similar time period. Trauma, foreign bodies, abscess/cellulitis of head and neck, airway compromise, acute vertigo and epistaxis constitute majority of Emergency calls for an Otolaryngology resident. The Fig. 1 shows the distribution of cases attended in Emergency department by Otolaryngology resident. It has been observed that patients presenting with foreign bodies, trauma, abscess/cellulitis and Giddiness have increased. This might be due to increased referrals because of shut down of services by smaller hospitals.

\section{Conclusion}

The balance of Otolaryngology resident training has shifted towards remote internet based academic sessions during COVID times, due to decrease in core patient related services. These changes are forced upon us due to the 
pandemic and the competency levels desired for residents have also changed. It is incumbent upon institutions to tackle the forced change and introduce novel methods to offset the loss of traditional teaching methods.

\section{Funding None.}

\section{Declarations}

Conflict of interest The authors declare that they have no conflict of interest.

\section{References}

1. De Luca P, Colacurcio V, De Bonis E, Petrosino M, Bisogno A, Troisi D et al (2020) Impact of the COVID-19 pandemic on otolaryngology residency: a real-life experience. Ear Nose Throat J 99(9):563-564

2. Crosby DL, Sharma A (2020) Insights on otolaryngology residency training during the COVID-19 pandemic. Otolaryngol Neck Surg 163(1):38-41

3. Sekar R, Alexander A, Ganesan S (2020) Challenges faced by an otolaryngology resident during Covid-19 Pandemic: an Indian perspective. Indian J Otolaryngol Head Neck Surg 1:1-4

Publisher's Note Springer Nature remains neutral with regard to jurisdictional claims in published maps and institutional affiliations. 